\title{
Burden of migraine in Finland: multimorbidity and phenotypic disease networks in occupational healthcare
}

\author{
Minna A. Korolainen ${ }^{1 *}$ DD, Samuli Tuominen², Samu Kurki ${ }^{3}$, Mariann I. Lassenius ${ }^{2}$, liro Toppila², Timo Purmonen?',
} Jaana Santaholma ${ }^{1}$ and Markku Nissilä ${ }^{3}$

\begin{abstract}
Background: Migraine is a complex neurological disorder with high co-existing morbidity burden. The aim of our study was to examine the overall morbidity and phenotypic diseasome for migraine among people of working age using real world data collected as a part of routine clinical practice.

Methods: Electronic medical records (EMR) of patients with migraine $(n=17,623)$ and age- and gender matched controls $(n=17,623)$ were included in this retrospective analysis. EMRs were assessed for the prevalence of ICD-10 codes, those with at least two significant phi correlations, and a prevalence $>2.5 \%$ in migraine patients were included to phenotypic disease networks (PDN) for further analysis. An automatic subnetwork detection algorithm was applied in order to cluster the diagnoses within the PDNs. The diagnosis-wise connectivity based on the PDNs was compared between migraine patients and controls to assess differences in morbidity patterns.

Results: The mean number of diagnoses per patient was increased 1.7-fold in migraine compared to controls. Altogether 1337 different ICD-10 codes were detected in EMRs of migraine patients. Monodiagnosis was present in 1\% and 13\%, and the median number of diagnoses was 12 and 6 in migraine patients and controls. The number of significant phi-correlations was 2.3-fold increased, and cluster analysis showed more clusters in those with migraine vs. controls (9 vs. 6). For migraine, the PDN was larger and denser and exhibited one large cluster containing fatigue, respiratory, sympathetic nervous system, gastrointestinal, infection, mental and mood disorder diagnoses. Migraine patients were more likely affected by multiple conditions compared to controls, even if no notable differences in morbidity patterns were identified through connectivity measures. Frequencies of ICD-10 codes on a three character and block level were increased across the whole diagnostic spectrum in migraine.
\end{abstract}

Conclusions: Migraine was associated with an increased multimorbidity, evidenced by multiple different approaches in the study. A systematic increase in the morbidity across the whole spectrum of ICD-10 coded diagnoses, and when interpreting PDNs, were detected in migraine patients. However, no specific diagnoses explained the morbidity. The results reflect clinical praxis, but also undoubtedly, the pathophysiological phenotypes related to migraine, and emphasize the importance of better understanding migraine-related morbidity.

Keywords: Diseasome, Multimorbidity, Migraine, Networks, ICD-10, Occupational healthcare

\footnotetext{
* Correspondence: Minna.Korolainen@novartis.com

${ }^{1}$ Novartis Finland Oy, Espoo, Finland

Full list of author information is available at the end of the article
}

(c) The Author(s). 2020 Open Access This article is distributed under the terms of the Creative Commons Attribution 4.0 International License (http://creativecommons.org/licenses/by/4.0/), which permits unrestricted use, distribution, and reproduction in any medium, provided you give appropriate credit to the original author(s) and the source, provide a link to the Creative Commons license, and indicate if changes were made. 


\section{Introduction}

Multimorbidity, defined as the co-occurrence of two or more diseases or conditions in an individual, has been described in migraine [1-4]. Although multimorbidity generally increases with age, comorbidities are present already in pediatric migraine [5]. The importance of multimorbidity is emphasized by an increase in health and social care expenditure per additional morbidity [6]. Global burden of disease repeatedly identifies migraine as one of the top conditions resulting in years lived with disability, likely attributable to the multimorbid strain on individuals $[7,8]$.

Physical and mental comorbidity in migraine has been examined extensively, and yet remain intricate. The evidence is mostly based on pairwise comparisons or examination of targeted conditions. A large number of migraine-associated comorbidities have been identified including asthma and allergies, psychiatric, cardiovascular, cerebrovascular, gastrointestinal, musculoskeletal disorders, as well as other neurological and pain-related disorders [9-19]. Moreover, several symptoms related to migraine attacks such as sensitivity to light, sound and odours also occur to some extent between migraine attacks. This may be explained by a lower general discomfort threshold in response to sensory stimuli, leading to enhanced perception of both painful and non-painful sensations [20]. Migraine is acknowledged as a complex genetic disorder that runs in families. Current evidence from genome-wide association studies mainly point at vascular function and metal ion channel activity involvement in the pathophysiology, whereas less genes linking to neuronal function and ion channel activity have been found [21].

The prevalence of multimorbidity has been estimated to be $13-83 \%$, and multimorbidity, as such, is understudied and underpublished [22]. Networks underlying multimorbidity are complex. Sturmberg and colleagues argued multimorbidity being "the manifestation of interconnected physiological network processes within an individual in his or her socio-cultural environment" [23]. This very well describes the complexity in understanding holistic and personalised disease patterns in individuals when these networks include multiple -omics, neuroendocrine, immune cell and mitochondrial energy-related elements as well as social, environmental, and healthcare networks.

Human diseasome is thought to comprehend disease genome and disease phenome. Phenotypic disease networks (PDN) provide a holistic view over a condition and the related multimorbidity. Previously PDNs have been used to study the multimorbidity patterns underlying depression as well as heart failure, migraine, diabetes and dementia in elderly patients [3, 24]. There migraine has been shown to be comorbid with for example depression, diabetes mellitus, and irritable bowel syndrome. Disease progression has been studied by assessing the directionality of PDNs as well as the association between the lethality and the connectivity in a PDN of a given condition [25]. The results suggested that patients are more likely to be affected by conditions that are close to the conditions already affecting them in a PDN, however, migraine was not present in the analyses.

We have previously shown that migraine was associated with a 1.7 -fold increase in healthcare resource utilization and 1.8-fold increase sick-leave days when compared to age and gender matched controls. Less than $10 \%$ of visits and sick-leaves were linked directly to migraine, and we detected that some of the diagnoses such as depression and anxiety were more frequent among migraine patients when compared to age- and gender-matched controls. Notably, the disease burden including the frequency of some additional co-existing morbidities increased by failing prophylactic treatments [26].

The aim of the current study was to further investigate comprehensive patterns of morbidity based on ICD-10 coded phenotypic diseasomes in migraine patients compared to age- and gender-matched controls. Migraine was associated with significant increase in overall morbidity seen both as increased multimorbidity across the ICD-10 coded diagnostic spectrum and in the larger PDN, in which diagnoses clustered differentially between migraine patients and controls. These findings strongly point at a significant multimorbidity among migraine patients that may reflect the polygenic nature of migraine but also complex representation of migraine symptoms in ICD-10 coded clinical praxis.

\section{Material and methods}

This retrospective register study included migraine patients using occupational healthcare with a G43* diagnosis in the electronic medical records (EMR) between 1st January 2012 and 31st December 2017 at the private healthcare provider Terveystalo. Altogether, 17,623 of the patients had migraine according to ICD-10 code $\left(\mathrm{G} 43^{*}\right.$, on a three-character level) and were included to this study. A one-to-one, age- and gender-matched control population without migraine was created. For each migraine patient a control patient was randomly chosen based on gender and birth date from the database. No subjects were chosen twice for the control group [Controls: $N=17,623,76,804$ patient-years, average age 38.9 years, $78.9 \%$ female; migraine patients: $N=17,623,51$, 396 patient-years, average age 38.9 years, $78.9 \%$ female]. For both migraine patients and controls, diagnoses were assessed from the EMR during the study period, 
irrespective of their timing in relation to the migraine diagnosis. Controls were used as reference for comorbidity estimations. The study cohort has been described in detail previously [26].

Disease networks and clustering were done at an ICD10 code three-character level e.g. H01*. Diagnostic codes with a prevalence of $2.5-20 \%$ in migraineurs were included in the network analysis. The most common diagnostic codes (prevalence $>20 \%$ in the migraine patients) were excluded from the network analyses but assessed separately, as these high prevalence morbidities would have been the main drivers for cluster formations, which would have resulted in yielding worse clusters as assessed by modularity.

Phi-correlations were calculated between 205 and 105 diagnostic codes in migraine patients and controls, respectively. The phi-correlations and the statistical significance were calculated following Hidalgo et al. 2009 [25]. Phi correlation was chosen over the relative risk ratio due to more convenient numeric properties i.e. approximate normal distribution without requiring any transformations. Briefly, phi correlation is calculated like the regular Pearson correlation, but between two binary variables, here if a patient was or was not recorded with a given diagnosis code. Thus, the possible values of the phi correlation range from -1 to 1 . Phi correlation -1 between two diagnosis codes mean that exactly the patients that were recorded with the diagnosis code 1 were not recorded with the diagnosis code 2 , and conversely for the diagnosis code 2 . Phi correlation 1 means that exactly the same patients were recorded with both diagnosis code 1 and 2. Phi correlation 0 means that there was no correlation between the diagnosis codes. The significance level 0.05 was used throughout and the significance was calculated as in Hidalgo et al. 2009 [25]. Furthermore, co-existing morbidities that significantly correlated with only one other morbidity were excluded from clustering and the network visualizations.

An automatic cluster detection method called the Walktrap-algorithm was applied in order to distinguish more closely related subsets of potential comorbidities [27]. The Walktrap-algorithm uses short random walks within the network weighted by the phi-correlations. One random walk consists of first selecting a diagnosis code at random and then again randomly selecting another diagnosis code that has a phi correlation with the current diagnosis code. Diagnosis codes with higher phi correlations are more likely to be selected as the second diagnosis code. The second selected diagnosis code becomes the current diagnosis code completing one step. These steps were repeated a specified number of times which is called a random walk. Diagnosis codes that are often part of the same random walk clustered together. Random walk lengths of 4 were used in this study.

The network visualizations utilize a spring layout where comorbidities with higher phi-correlations are placed closer to each other. The modularity and four diagnosis code - wise centrality measures, namely the degree, betweenness, closeness and strength, were calculated, reported, and visualized [28]. Explanations of the calculation and interpretation of the centrality measures are included in Appendix 1 [27, 28]. The degree distributions of migraineurs and controls were compared using a scatterplot and by regressing the number of significant phi-correlations in migraineurs on the controls. Outliers were detected using the mean-shift test at significance level 0.05 with Bonferroni correction.

The number of distinct diagnoses per person was assessed from ICD-10 codes for controls and patients with migraine. The frequency of patients per number of diagnosis codes for diagnosis codes included in the PDNs were reported. Overall diagnoses were further assessed at a block level (e.g. H53-H54, visual disturbances and blindness) in migraine patients vs. controls. Blocks with prevalence over $2 \%$ in migraine patients and fold change of at least 1.5 were reported. The prevalence differences between migraine patients and controls were tested with Chi-squared test at significance level 0.05 . Baseline characteristics are presented at the time of the first G43* diagnosis.

All analyses were conducted using R: A language and environment for statistical computing, version 3.5.2. The network analyses and visualizations used the ggraph and igraph packages available on the Comprehensive R Archive Network (CRAN).

\section{Results}

Patients with migraine were on average 39 years, and the majority were women (Table 1 ). Prophylactic migraine medication was prescribed to $13 \%$ of the cohort, acute migraine medication to $37 \%$ and $51 \%$ had no migraine prescriptions from the occupational healthcare [26]. Controls were lacking G43*diagnosis,

Table 1 Baseline characteristics of the migraine patients and controls

\begin{tabular}{lll}
\hline & Controls & Migraine $\left(\mathrm{G} 43^{*}\right)$ \\
\hline Follow-up time, median (min-max) & $5.19(0.11-6.00)$ & $2.84(0.09-6.00)$ \\
$\mathrm{N}$ & 17,623 & 17,623 \\
Female, \% & $78.9 \%$ & $78.9 \%$ \\
Age, mean & $39(15-77)$ & $39(15-77)$ \\
\hline
\end{tabular}


and matched based on age and gender. The median follow-up time was 2.5 years longer for controls than for migraine patients. The study provides new insight on increased multimorbidity across all diagnosis codes in migraine and shows that diagnoses cluster differentially between migraine patients and controls in phenotypic disease networks.

Altogether 1337 different ICD-10 codes were detected in EMRs, but all were not included in the further analyses due to low abundancy. The mean number of diagnoses per patient was increased 1.7fold in migraine compared to controls. The median number of distinct diagnoses per person was 12 for migraine patients and 6 for controls. In the migraine patients $1.0 \%$ had only one diagnosis (i.e. monomorbidity) whereas $12.8 \%$ of controls had only one diagnosis. A histogram with the frequency of patients or controls per number of distinct diagnoses per person is presented in Fig. 1.

The individual diagnoses that are present in comorbidity networks are shown in in Table 2 and the PDNs in Fig. 2. Moreover, the frequencies for each of these diagnoses exhibited a significant increase among migraine patients when compared to controls (Table 2). The number of significant phi-correlations $(p<0.05)$ was greater in patients with migraine than among controls (4752 vs. 2804). There were 197 potential co-existing morbidities in migraine patients and 148 morbidities in controls with at least two significant phi-correlations. The median number of significant phi-correlations per diagnosis code was 12 and 9 for migraine patients and controls, respectively.

Altogether nine clusters were formed for migraine patients (Fig. 2). A large cluster M1 shown in Fig. 2, emerged where the diagnoses that were linked with each other included mental disorders (e.g. anxiety F41, fatigue R53, stress-related disorder F43) respiratory disorders (e.g. asthma J45, cough R05), sympathetic nervous system disorders (e.g. disorders of the vestibular system $\mathrm{H} 81$, disorders of the lacrimal system H04), infections (e.g. J03 acute tonsillitis, R50 fever), and gastrointestinal problems (e.g. IBS K58, dyspepsia K30, gastritis and duodenitis K29). Another cluster M2 was formed of musculoskeletal and connective tissue, nerve root and menopausal diagnoses. The other seven clusters consisted of more homogeneous diagnostic codes that could be explained by single nominators including injuries (M3), ear infections (M4), dermatitis (M6), acne/rosacea (M7), eye (M8), skin (M5), and urogenital disorders (M9).

The comorbidity network was smaller in controls consisting of six clusters (vs. nine in migraine). Further differences included clusters $\mathrm{C} 1, \mathrm{C} 2$, and $\mathrm{C} 4$ that consisted of more heterogeneous diagnosis codes as well as clusters with diagnostic codes that could be identified by single nominators such as injuries (C3), dermatitis (C6) and skin disorders (C5) (Fig. 2 and Table 2).

Multiple descriptive network measures were calculated from the PDNs for migraine and controls. See Appendix 1 for a summary on the calculation and interpretation of these measures. The modularities related to the clustering were 0.23 and 0.31 for the migraine and controls, respectively, indicating that the obtained clusterings were sensible. The difference in modularity values likely reflects the increased overall morbidity in migraine patients leading to higher phi-correlations and greater overall

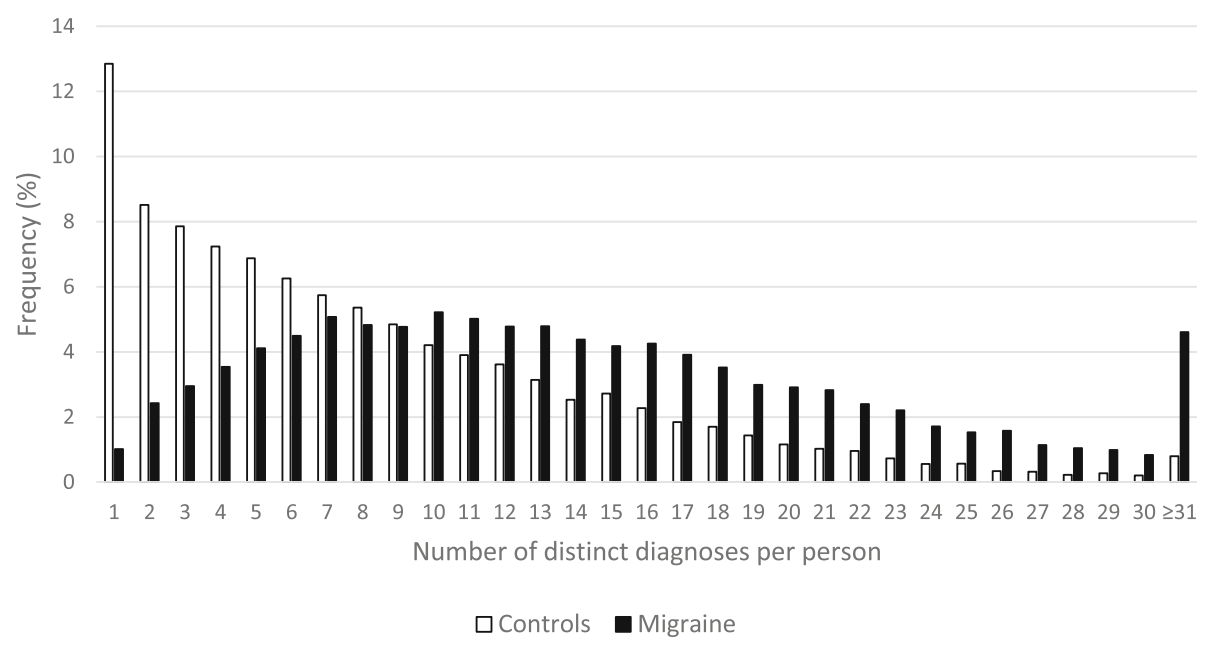

Fig. 1 The frequency of patients per distinct number of diagnoses per patient among patients with migraine and controls 
Table 2 List of diagnosis clusters from Fig. 2 sorted by their presence in migraine patients (2.5-20\% prevalence) and compared to controls. Fold change column shows the prevalence in migraineurs divided by the prevalence in controls. All fold changes are greater than 1 indicating that migraine is associated with higher multimorbidity compared to controls. Due to high sample size, the difference in prevalence between migraineurs and controls is statistically significant $(p<0.001)$ for each diagnosis

\begin{tabular}{|c|c|c|c|c|c|c|}
\hline Code & Migraine cluster & Control cluster & Name & M (\%) & C (\%) & Fold change \\
\hline A08 & M1 & N/A & Viral and other specified intestinal infections & 4.10 & 1.80 & 2.28 \\
\hline E03 & M1 & C4 & Other hypothyroidism & 5.20 & 3.30 & 1.58 \\
\hline F32 & M1 & C4 & Depressive episode & 13.00 & 7.10 & 1.83 \\
\hline F33 & M1 & N/A & Recurrent depressive disorder & 4.80 & 2.50 & 1.92 \\
\hline F41 & M1 & C4 & Other anxiety disorders & 14.10 & 7.90 & 1.78 \\
\hline $\mathrm{F} 43$ & M1 & C4 & Reaction to severe stress, and adjustment disorders & 17.40 & 9.90 & 1.76 \\
\hline F51 & M1 & C4 & Nonorganic sleep disorders & 17.40 & 8.80 & 1.98 \\
\hline G44 & M1 & C4 & Other headache syndromes & 19.50 & 5.90 & 3.31 \\
\hline G47 & M1 & N/A & Sleep disorders & 4.60 & 2.40 & 1.92 \\
\hline $\mathrm{HO4}$ & M1 & C1 & Disorders of lacrimal system & 4.10 & 2.50 & 1.64 \\
\hline $\mathrm{H} 10$ & M1 & C1 & Conjunctivitis & 16.20 & 11.00 & 1.47 \\
\hline H81 & M1 & N/A & Disorders of vestibular function & 4.50 & 2.30 & 1.96 \\
\hline 149 & M1 & C1 & Other cardiac arrhythmias & 6.60 & 3.90 & 1.69 \\
\hline 184 & M1 & $\mathrm{C} 1$ & Haemorrhoids & 5.30 & 3.20 & 1.66 \\
\hline J00 & M1 & $\mathrm{C} 1$ & Acute nasopharyngitis [common cold] & 6.00 & 3.60 & 1.67 \\
\hline J02 & M1 & C1 & Acute pharyngitis & 15.50 & 9.40 & 1.65 \\
\hline J03 & M1 & $\mathrm{C} 1$ & Acute tonsillitis & 9.90 & 6.50 & 1.52 \\
\hline J04 & M1 & C1 & Acute laryngitis and tracheitis & 10.20 & 5.90 & 1.73 \\
\hline J11 & M1 & C1 & Influenza, virus not identified & 6.10 & 3.70 & 1.65 \\
\hline$J 22$ & M1 & C2 & Unspecified acute lower respiratory infection & 4.50 & 2.60 & 1.73 \\
\hline J30 & M1 & $\mathrm{C} 1$ & Vasomotor and allergic rhinitis & 10.80 & 6.50 & 1.66 \\
\hline J31 & M1 & C1 & Chronic rhinitis, nasopharyngitis and pharyngitis & 5.00 & 2.90 & 1.72 \\
\hline J45 & M1 & C1 & Asthma & 8.40 & 5.40 & 1.56 \\
\hline K21 & M1 & C1 & Gastro-oesophageal reflux disease & 7.10 & 4.00 & 1.78 \\
\hline K29 & M1 & N/A & Gastritis and duodenitis & 3.60 & 1.80 & 2.00 \\
\hline K30 & M1 & C1 & Dyspepsia & 6.00 & 3.00 & 2.00 \\
\hline K58 & M1 & C1 & Irritable bowel syndrome & 4.80 & 2.50 & 1.92 \\
\hline K59 & M1 & N/A & Other functional intestinal disorders & 3.60 & 1.90 & 1.89 \\
\hline L03 & M1 & C2 & Cellulitis & 4.60 & 2.70 & 1.70 \\
\hline ROO & M1 & N/A & Abnormalities of heart beat & 3.30 & 1.80 & 1.83 \\
\hline R05 & M1 & C1 & Cough & 8.90 & 5.90 & 1.51 \\
\hline R06 & M1 & C1 & Abnormalities of breathing & 6.30 & 3.80 & 1.66 \\
\hline R07 & M1 & C1 & Pain in throat and chest & 11.20 & 6.50 & 1.72 \\
\hline R11 & M1 & N/A & Nausea and vomiting & 3.90 & 1.60 & 2.44 \\
\hline R42 & M1 & C4 & Dizziness and giddiness & 7.10 & 3.40 & 2.09 \\
\hline R50 & M1 & N/A & Fever of other and unknown origin & 4.90 & 2.70 & 1.81 \\
\hline R51 & M1 & C4 & Headache & 18.10 & 4.10 & 4.41 \\
\hline R52 & M1 & N/A & Pain, not elsewhere classified & 3.20 & 1.70 & 1.88 \\
\hline R53 & M1 & C4 & Malaise and fatigue & 12.20 & 6.70 & 1.82 \\
\hline S13 & M1 & N/A & Dislocation, sprain and strain of joints and ligaments at neck level & 2.60 & 1.30 & 2.00 \\
\hline T78 & M1 & N/A & Adverse effects, not elsewhere classified & 3.20 & 1.80 & 1.78 \\
\hline Z01 & M1 & C1 & Other special examinations and investigations of persons without & 19.80 & 14.10 & 1.40 \\
\hline
\end{tabular}


Table 2 List of diagnosis clusters from Fig. 2 sorted by their presence in migraine patients (2.5-20\% prevalence) and compared to controls. Fold change column shows the prevalence in migraineurs divided by the prevalence in controls. All fold changes are greater than 1 indicating that migraine is associated with higher multimorbidity compared to controls. Due to high sample size, the difference in prevalence between migraineurs and controls is statistically significant $(p<0.001)$ for each diagnosis (Continued)

\begin{tabular}{|c|c|c|c|c|c|c|}
\hline Code & Migraine cluster & Control cluster & Name & M (\%) & C (\%) & Fold change \\
\hline & & & complaint or reported diagnosis & & & \\
\hline Z03 & M1 & C1 & $\begin{array}{l}\text { Medical observation and evaluation for suspected diseases } \\
\text { and conditions }\end{array}$ & 6.20 & 3.60 & 1.72 \\
\hline Z71 & M1 & $\mathrm{C} 1$ & $\begin{array}{l}\text { Persons encountering health services for other counselling } \\
\text { and medical advice, not elsewhere classified }\end{array}$ & 15.60 & 10.00 & 1.56 \\
\hline Z73 & M1 & N/A & Problems related to life-management difficulty & 3.20 & 1.50 & 2.13 \\
\hline E78 & M2 & C2 & Disorders of lipoprotein metabolism and other lipidaemias & 4.40 & 3.20 & 1.38 \\
\hline G56 & M2 & $\mathrm{C} 2$ & Mononeuropathies of upper limb & 4.50 & 2.90 & 1.55 \\
\hline 110 & M2 & C2 & Essential (primary) hypertension & 11.30 & 8.70 & 1.30 \\
\hline J18 & M2 & N/A & Pneumonia, organism unspecified & 3.20 & 2.50 & 1.28 \\
\hline M19 & M2 & N/A & Other arthrosis & 2.50 & 1.80 & 1.39 \\
\hline M25 & M2 & C2 & Other joint disorders & 19.60 & 12.80 & 1.53 \\
\hline M51 & M2 & $\mathrm{C} 2$ & Other intervertebral disc disorders & 6.70 & 4.10 & 1.63 \\
\hline M62 & M2 & N/A & Other disorders of muscle & 3.50 & 1.80 & 1.94 \\
\hline M65 & M2 & C2 & Synovitis and tenosynovitis & 7.00 & 4.60 & 1.52 \\
\hline M67 & M2 & N/A & Other disorders of synovium and tendon & 3.20 & 2.10 & 1.52 \\
\hline M70 & M2 & C2 & Soft tissue disorders related to use, overuse and pressure & 8.00 & 4.90 & 1.63 \\
\hline M72 & M2 & C2 & Fibroblastic disorders & 5.40 & 3.60 & 1.50 \\
\hline M75 & M2 & C2 & Shoulder lesions & 19.90 & 13.30 & 1.50 \\
\hline M76 & M2 & C2 & Enthesopathies of lower limb, excluding foot & 4.70 & 3.10 & 1.52 \\
\hline M77 & M2 & C2 & Other enthesopathies & 15.30 & 9.90 & 1.55 \\
\hline N95 & M2 & $\mathrm{C} 1$ & Menopausal and other perimenopausal disorders & 7.90 & 5.20 & 1.52 \\
\hline S43 & M2 & N/A & $\begin{array}{l}\text { Dislocation, sprain and strain of joints and ligaments of shoulder } \\
\text { girdle }\end{array}$ & 3.40 & 2.10 & 1.62 \\
\hline Z10 & M2 & $\mathrm{C} 1$ & Routine general health check-up of defined subpopulation & 10.60 & 8.90 & 1.19 \\
\hline M17 & M3 & C2 & Gonarthrosis [arthrosis of knee] & 4.50 & 3.50 & 1.29 \\
\hline M23 & M3 & C2 & Internal derangement of knee & 7.10 & 4.80 & 1.48 \\
\hline S60 & M3 & $\mathrm{C} 3$ & Superficial injury of wrist and hand & 5.40 & 3.10 & 1.74 \\
\hline S61 & M3 & N/A & Open wound of wrist and hand & 4.00 & 2.90 & 1.38 \\
\hline S63 & M3 & C3 & $\begin{array}{l}\text { Dislocation, sprain and strain of joints and ligaments at wrist and } \\
\text { hand level }\end{array}$ & 5.20 & 3.40 & 1.53 \\
\hline S80 & M3 & $\mathrm{C} 2$ & Superficial injury of lower leg & 4.50 & 3.00 & 1.50 \\
\hline S83 & M3 & C2 & Dislocation, sprain and strain of joints and ligaments of knee & 6.20 & 4.50 & 1.38 \\
\hline S90 & M3 & N/A & Superficial injury of ankle and foot & 4.00 & 2.30 & 1.74 \\
\hline S93 & M3 & $\mathrm{C} 2$ & $\begin{array}{l}\text { Dislocation, sprain and strain of joints and ligaments at ankle and } \\
\text { foot level }\end{array}$ & 8.60 & 5.60 & 1.54 \\
\hline H60 & M4 & $\mathrm{C} 1$ & Otitis externa & 6.90 & 4.50 & 1.53 \\
\hline H65 & M4 & N/A & Nonsuppurative otitis media & 3.40 & 2.10 & 1.62 \\
\hline H66 & M4 & $\mathrm{C} 1$ & Suppurative and unspecified otitis media & 8.80 & 5.90 & 1.49 \\
\hline H92 & M4 & N/A & Otalgia and effusion of ear & 3.10 & 1.80 & 1.72 \\
\hline D22 & M5 & C5 & Melanocytic naevi & 9.90 & 7.10 & 1.39 \\
\hline D23 & M5 & C5 & Other benign neoplasms of skin & 4.40 & 3.20 & 1.38 \\
\hline $\mathrm{H} 52$ & M5 & $\mathrm{C} 1$ & Disorders of refraction and accommodation & 6.90 & 4.00 & 1.73 \\
\hline
\end{tabular}


Table 2 List of diagnosis clusters from Fig. 2 sorted by their presence in migraine patients (2.5-20\% prevalence) and compared to controls. Fold change column shows the prevalence in migraineurs divided by the prevalence in controls. All fold changes are greater than 1 indicating that migraine is associated with higher multimorbidity compared to controls. Due to high sample size, the difference in prevalence between migraineurs and controls is statistically significant $(p<0.001)$ for each diagnosis (Continued)

\begin{tabular}{|c|c|c|c|c|c|c|}
\hline Code & Migraine cluster & Control cluster & Name & M (\%) & C (\%) & Fold change \\
\hline $\mathrm{H} 53$ & M5 & N/A & Visual disturbances & 4.20 & 1.40 & 3.00 \\
\hline L82 & M5 & C5 & Seborrhoeic keratosis & 3.90 & 2.60 & 1.50 \\
\hline R23 & M5 & N/A & Other skin changes & 2.80 & 1.70 & 1.65 \\
\hline B35 & M6 & C6 & Dermatophytosis & 4.20 & 3.20 & 1.31 \\
\hline LO2 & M6 & $\mathrm{C} 2$ & Cutaneous abscess, furuncle and carbuncle & 4.80 & 3.10 & 1.55 \\
\hline L08 & M6 & N/A & Other local infections of skin and subcutaneous tissue & 3.00 & 2.00 & 1.50 \\
\hline L20 & M6 & C6 & Atopic dermatitis & 5.80 & 4.20 & 1.38 \\
\hline L30 & M6 & C6 & Other dermatitis & 8.90 & 6.50 & 1.37 \\
\hline L50 & M6 & N/A & Urticaria & 3.50 & 2.30 & 1.52 \\
\hline R22 & M6 & C5 & $\begin{array}{l}\text { Localized swelling, mass and lump of skin and subcutaneous } \\
\text { tissue }\end{array}$ & 5.90 & 4.00 & 1.48 \\
\hline L70 & M7 & N/A & Acne & 4.10 & 2.40 & 1.71 \\
\hline L71 & M7 & N/A & Rosacea & 3.00 & 1.60 & 1.88 \\
\hline $\mathrm{HOO}$ & M8 & N/A & Hordeolum and chalazion & 3.30 & 2.00 & 1.65 \\
\hline H01 & M8 & C6 & Other inflammation of eyelid & 4.10 & 2.50 & 1.64 \\
\hline N30 & M9 & C4 & Cystitis & 13.50 & 9.00 & 1.50 \\
\hline N39 & M9 & C4 & Other diseases of urinary system & 6.70 & 4.40 & 1.52 \\
\hline N64 & M9 & N/A & Other disorders of breast & 2.50 & 1.60 & 1.56 \\
\hline N76 & M9 & C4 & Other inflammation of vagina and vulva & 5.70 & 3.90 & 1.46 \\
\hline N92 & M9 & C4 & Excessive, frequent and irregular menstruation & 9.20 & 6.90 & 1.33 \\
\hline N94 & M9 & N/A & $\begin{array}{l}\text { Pain and other conditions associated with female genital organs } \\
\text { and menstrual cycle }\end{array}$ & 3.10 & 1.60 & 1.94 \\
\hline Z00 & M9 & C4 & $\begin{array}{l}\text { General examination and investigation of persons without complaint } \\
\text { and reported diagnosis }\end{array}$ & 8.60 & 5.30 & 1.62 \\
\hline Z30 & M9 & C4 & Contraceptive management & 6.40 & 5.10 & 1.25 \\
\hline
\end{tabular}

connectivity in the PDN. Regressing the degrees pertaining to each comorbidity in migraine on the controls shows that the degree in controls predicts the degree in migraine patients well; on average one significant phi-correlation in controls implies 1.4 significant phi-correlations in migraine patients per comorbidity (regression slope 1.4, $p<0.001$, Fig. 3). However, three outliers to this are detected using the mean-shift outlier test, namely F43 (reaction to severe stress, and adjustment disorders), G44 (other headache syndromes), and R51 (headache) (Bonferroni corrected $p$-values $<0.001$ for all three diagnosis codes). Across the centrality measures comorbidities in migraine patients show increased connectivity indicating that a patient is more likely to be affected by multiple conditions (Fig. 4). The betweenness measure in Fig. 4 shows clearest differences between migraine and controls at F-codes, M75 (shoulder lesions) and N95 (menopausal and other perimenopausal disorders) and R-codes.

The 101 diagnostic codes that were present with a frequency of $2.5 \%-20 \%$ in migraine patients and had at least 2 significant phi-correlations included in the PDNs, exhibited significantly higher prevalence among migraine patients when compared to controls (Table 2). Figure 5 represents diagnoses with over $20 \%$ prevalence in migraine patients that were also significantly more frequent when compared to controls. The diagnostic code Z76 includes a group of heterogeneous sub-diagnostic codes related to health services and consisted mainly of the code Z76 as well as Z76.0 indicating issues of repeated prescriptions.

In addition, in order to get a more holistic view into multimorbidity, we also examined diagnoses on the block level. The whole spectrum of phenotypic diseasomes represented as ICD-10 blocks in migraine 


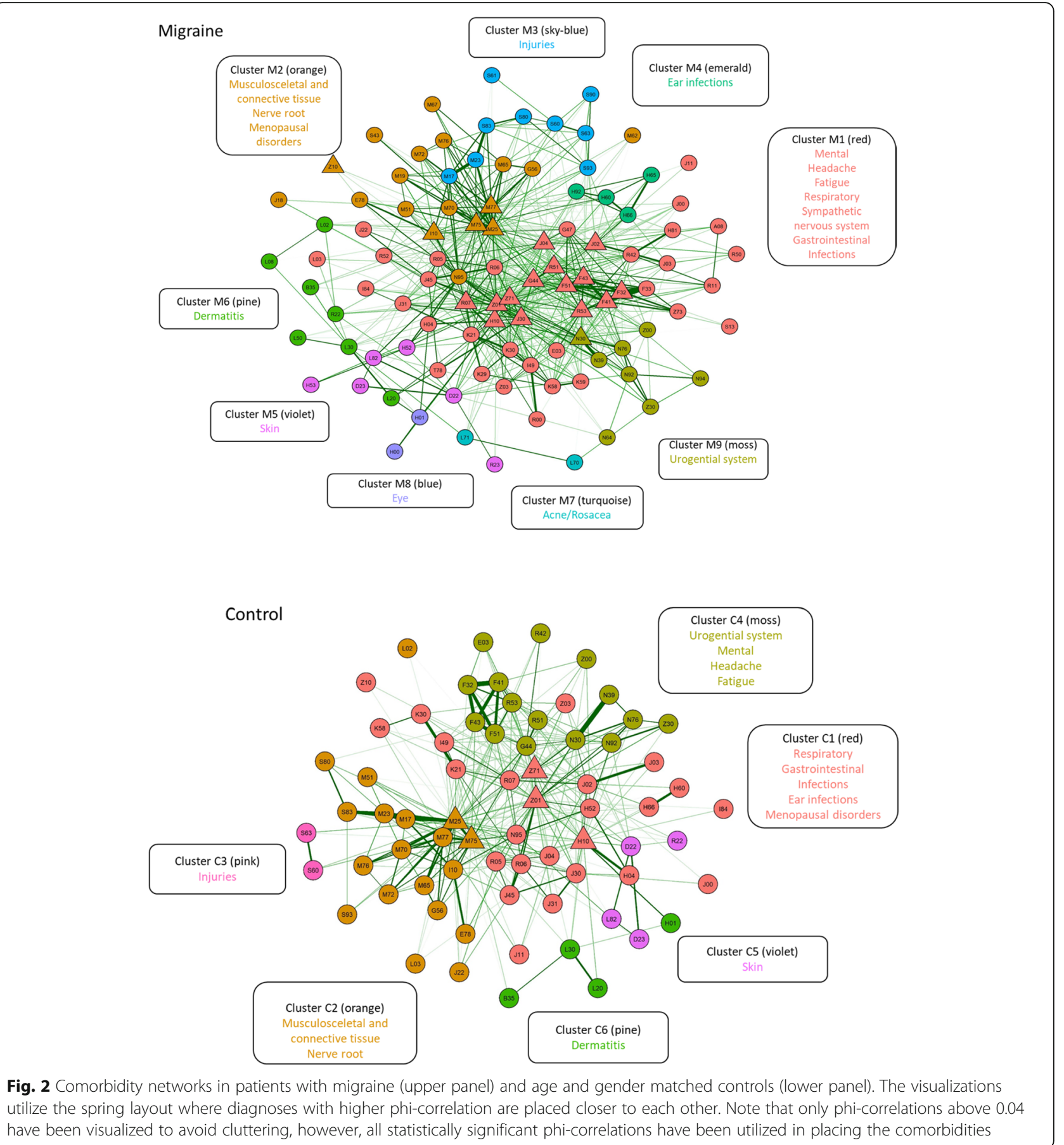

patients when compared to controls is visualized in Appendix 2. Migraine patients had an increase in overall diagnoses that were distributed across multiple ICD-10 code blocks. In Fig. 6 , the blocks with a $>2 \%$ prevalence in the migraine cohort and an $>1.5$-fold increase in prevalence compared to controls are presented. The largest enrichment was seen in visual disturbances, followed by episodic paroxysmal symptoms (G43* migraine code excluded). All together 56 blocks were enriched in patients with migraine (Fig. 6).

\section{Discussion}

With this study, we investigated the phenotypic diseasomes associated with migraine in an occupational healthcare setting using PDNs and frequencies of ICD-10 codes when compared to age- and gender- 


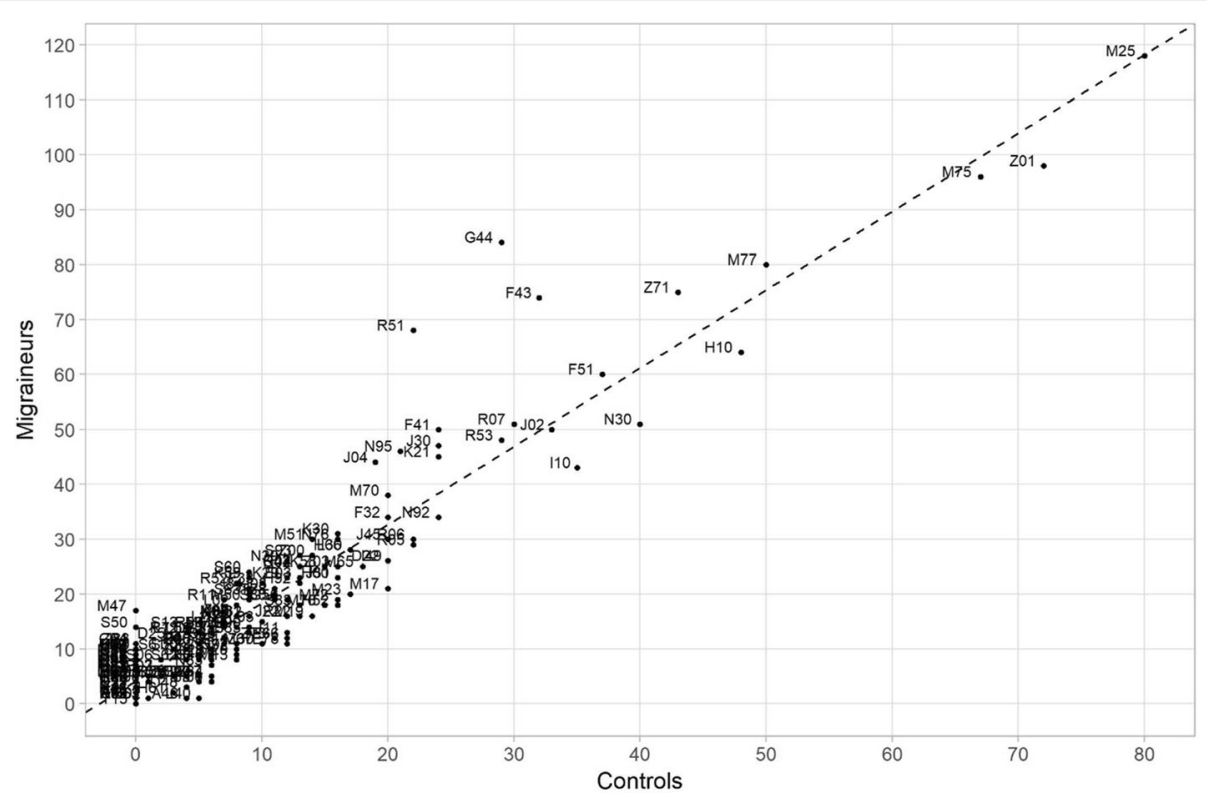

Fig. 3 Number of statistically significant phi-correlations per each comorbidity (degree) plotted in migraineurs vs controls. The dashed line represents the regression slope from regressing the number of significant phi-correlations in migraineurs on the controls. Slope $=1.43(p<0.001)$, intercept $=4.04(p<0.001)$, R-squared $=88.6 \%$. The fit shows that the number of significant phi-correlations per comorbidity in migraineurs is explained quite well by the respective number in controls, when the overall increase in morbidity in migraineurs is accounted for. However, visual assessment suggests 3 outliers: F43 (reaction to severe stress, and adjustment disorders), G44 (other headache syndromes), and R51 (headache). This is confirmed by a formal mean-shift outlier test (Bonferroni corrected $p$-values $<0.001$ for all three diagnosis codes)

matched control population. The most important results of the current study include 1) demonstrating that large datasets collected as a part of routine clinical praxis can be useful in naturally clustering diseasomes in an untargeted fashion; 2) diagnostic codes clustered differently into 9 and 6 clusters for migraine patients and controls, respectively; 3) the migraine PDN was larger and denser and exhibited one large cluster with functional-disorder-like symptoms including fatigue, respiratory, sympathetic nervous system, gastrointestinal, infection, mental and mood disorder diagnoses; 4) elucidating holistic and substantial multimorbidity for migraine seen as a holistic increase in prevalences of diagnoses across the whole ICD-10 coded diagnostic spectrum.

We have previously shown in the same population a substantial increase in healthcare visits and sick leaves for migraine patients compared to controls, and that less than $10 \%$ of these were linked to migraine in EMRs [26]. We further show here that this increase cannot be referred to a few driving morbidities but is associated with a generalized increase in multimorbidity. According to our best knowledge, this is the first untargeted approach to investigate the whole ICD-10 coded diseasome and PDN in migraine patients. Moreover, to our knowledge, this is the first study to include a matched control population for comparison when constructing PDNs. No other study has before elucidated this holistic and substantial multimorbidity for migraine. It is hoped that this study is followed by many more resulting in better understanding causes and consequences of migraine multimorbidity.

\section{Increased comorbidity based on phenotypic disease networks}

Not only were there more clusters forming for the migraine group, but also the clusters included different diagnostic groups than for controls. There were two larger clusters forming with multiple diagnostic classes (M1 and M2 in migraine as well as $\mathrm{C} 1$ and C4 for controls). It was challenging to find a single nominator for these groups and thus clusters have been named by numbers and indicated by colours.

Interestingly, in migraine, the largest cluster M1, consisted of many co-existing morbidities already suggested to be related to migraine. These disorders quite well mimic those also found in functional disorders, and this cluster could potentially be called "a functional disorder-like". As functional disorders have unknown causes but similar symptomology, it may be of interest to examine more underlying migraine in functional disorders or understand better the generalized symptoms apparently related to migraine [29]. Sympathetic disorders in cluster M1 and nerve root disorders in M2 represent an interesting 


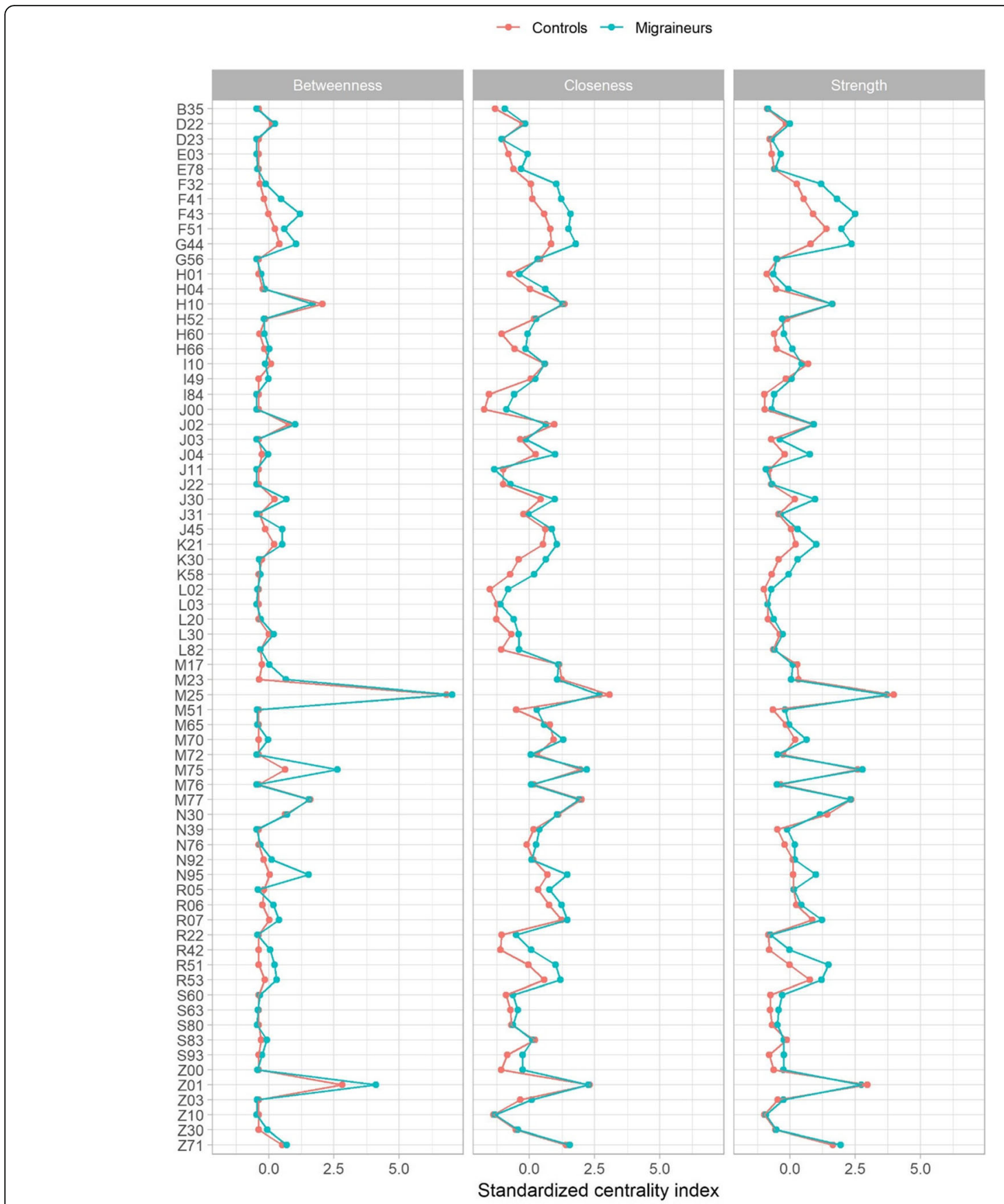

Fig. 4 Three centrality measures visualized for migraineurs and controls. These have been calculated from the PDNs in Fig. 2. Betweenness: how well a comorbidity connects other comorbidities; Closeness: how close the comorbidity is to other comorbidities in the PDN; Strength: the sum of phi correlations over all connected comorbidities. Note that instead of the raw centrality measure values, the standardized values (i.e. Z-scores) have been plotted. This enables the comparison of the values from the two PDNs. Generally, the centrality of the comorbidites in migraineurs follows the same pattern as in controls, however, there appears to be some differences in betweenness in F-codes, M75, N95 \& R-codes

common finding linking both clusters into the nervous system.

Increased connectivity in the PDN for migraine patients translates to higher morbidity meaning that a migraine patient is more likely to be affected by multiple conditions compared to controls. This was seen in the larger and denser PDN and the increased overall connectivity in all four centrality measures as well as 56 


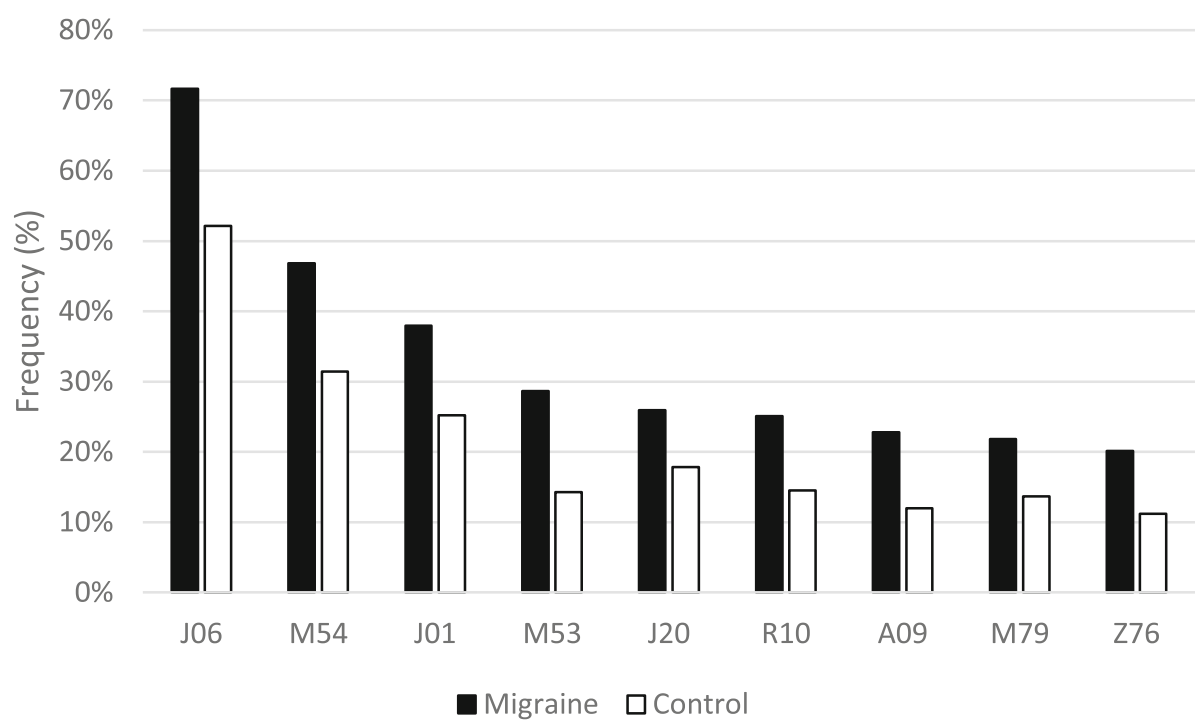

Fig. 5 The frequency of diagnoses with $>20 \%$ prevalence in the migraine patients. $J 06$ Acute upper respiratory infections of multiple and unspecified sites, M54Dorsalgia, J01 Acute sinusitis, M53 Other dorsopathies, J20 Acute bronchitis, R10 Abdominal and pelvic pain, A09 Diarrhoea and gastroenteritis of presumed infectious origin, M79 Other soft tissue disorders, Z76 Persons encountering health services in other circumstances

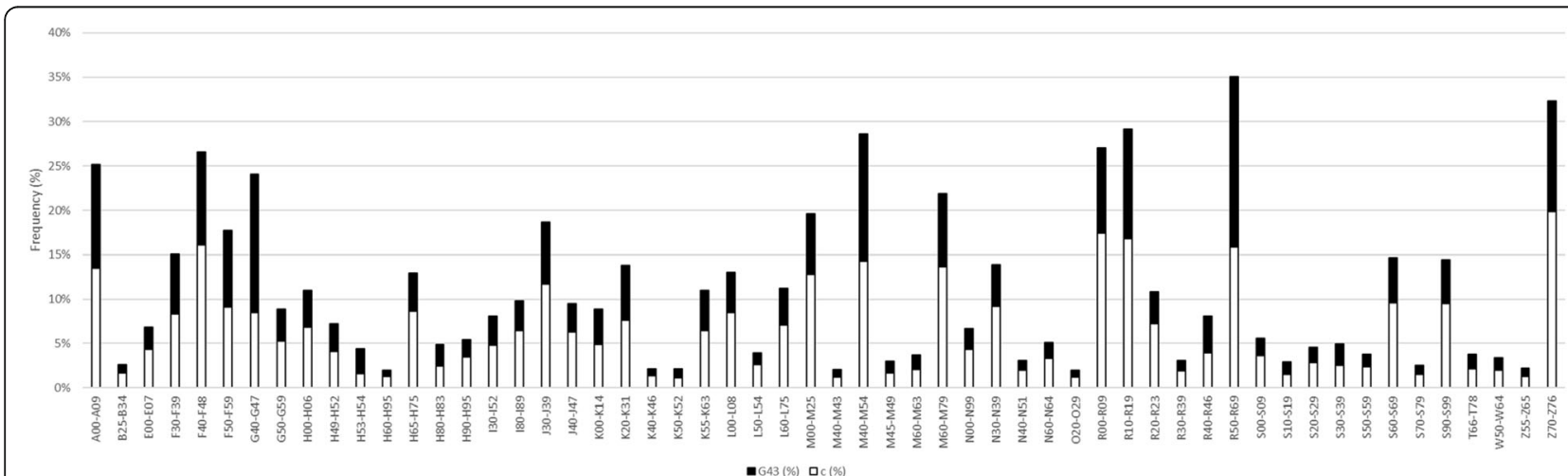

Fig. 6 Overall prevalence (\%) of diagnoses with $>2 \%$ prevalence in migraine patients with over 1.5 -fold increase in migraine patients (black) compared to controls (white), $p<0.001$ for all ICD-10 blocks. Bars are superimposed on each other. A00-A09 Intestinal infectious diseases, B25-B34 Other viral diseases, E00-E07 Disorders of thyroid gland, F30-F39 Mood [affective] disorders, F40-F48 Neurotic, stress-related and somatoform disorders, F50-F59 Behavioural syndromes associated with physiological disturbances and physical factors, G40-G47 Episodic and paroxysmal disorders, G50-G59 Nerve, nerve root and plexus disorders, H00-H06 Disorders of eyelid, lacrimal system and orbit, H49-H52 Disorders of ocular muscles, binocular movement, accommodation and refraction, H53-H54 Visual disturbances and blindness, H60-H95 Diseases of the ear and mastoid process, H65-H75 Diseases of middle ear and mastoid, H80-H83 Diseases of inner ear, H90-H95 Other disorders of ear, I30-152 Other forms of heart disease, 180-189 Diseases of veins, lymphatic vessels and lymph nodes, not elsewhere classified, J30-J39 Other diseases of upper respiratory tract, J40-J47 Chronic lower respiratory diseases, K00-K14 Diseases of oral cavity, salivary glands and jaws, K20-K31 Diseases of oesophagus, stomach and duodenum, K40-K46 Hernia, K55-K63 Other diseases of intestines, L00-L08 Infections of the skin and subcutaneous tissue, K50-K52 Noninfective enteritis and colitis, L50-L54 Urticaria and erythema, L60-L75 Disorders of skin appendages, M00-M25 Arthropathies, M40-M43 Deforming dorsopathies, M40-M54 Dorsopathies, M45-M49 Spondylopathies, M60-M63 Disorders of muscles, M60-M79 Soft tissue disorders, N00-N99 Diseases of the genitourinary system, N30-N39 Other diseases of urinary system, N40-N51 Diseases of male genital organs, N60-N64 Disorders of breast, R00-R09 Symptoms and signs involving the circulatory and respiratory systems, O20-O29 Other maternal disorders predominantly related to pregnancy, R10-R19 Symptoms and signs involving the digestive system and abdomen, R20-R23 Symptoms and signs involving the skin and subcutaneous tissue, R30-R39 Symptoms and signs involving the urinary system, R40-R46 Symptoms and signs involving cognition, perception, emotional state and behaviour, R50-R69 General symptoms and signs, S00-S09 Injuries to the head, S10-S19 Injuries to the neck, S20-S29 Injuries to the thorax, S30-S39 Injuries to the abdomen, lower back, lumbar spine and pelvis, S50-S59 Injuries to the elbow and forearm, S60-S69 Injuries to the wrist and hand, S70-S79 Injuries to the hip and thigh, S90-S99 Injuries to the ankle and foot, T66-T78 Other and unspecified effects of external causes, W50-W64 Exposure to animate mechanical forces, Z55-Z65 Persons with potential health hazards related to socioeconomic and psychosocial circumstances, Z70-Z76 Persons encountering health services in other circumstances 
blocks shown in Fig. 6 exhibiting 1.5-fold increase in migraine. Still, the betweenness measure hinted at some diagnoses being more central in migraineurs than in controls (F-codes, shoulder lesions, menopausal and other perimenopausal disorders, R-codes), which may indicate that these comorbidities are more important for the emergence of morbidity in migraine patients than in controls. It remains to be seen whether targeting some of these conditions may be beneficial in minimizing the multimorbidity among migraine patients.

In addition, outliers were detected in regressing the number of significant phi-correlations in migraineurs on controls, namely reaction to severe stress (F43), other headache syndromes (G44), and headache (R51). Whether some of the controls influencing the formation of cluster C4 (Fig. 2) represent undiagnosed migraine cases, needs further research. It may be of interest removing controls with R51 diagnosis as potential migraine to further evaluate whether and how this would influence clustering in non-migraine population.

Some more detailed diagnosis differences were evident in migraine patients. Unlike in controls, fatigue (R53) and healthcare visits regarding problems related to life management difficulty (Z73) correlated in the cluster $\mathrm{M} 1$ in migraine patients. The latter did not exhibit significant phi-correlations in controls and is thus missing from PDNs. The prevalence was over two-fold among migraine compared to controls although causes for this cannot be determined in this study.

Non-headache symptoms and pain are common in migraine although pathophysiological causes may be complex and are not well understood [30,31]. This was also seen in our study as frequencies of diagnoses for injuries, musculosceletal, connective tissue and nerve root disorders followed the same general pattern of increase in migraine despite clustering in the same fashion for both migraine patients and controls. Moreover, over 1.5-fold increase was detected for vestibular function, visual disturbances and dizziness (H81, H53, R42). It is possible that these changes may reflect migraine comorbidity related to balance and postural impairment as previously shown [14, 32-34]. Moreover, these may be linked to challenges in bodyboard control as detected in clinical practice for migraine patients.

Some diagnoses were in PDNs for migraine patients that were lacking in control networks; sleep disorders (G47) in cluster M1, acne (L70) and rosacea (L71) in cluster M7, and urticaria (L50) in cluster M6. The connection between migraine and sleep disorders has been recognized $[35,36]$. However, the connection of migraine and rosacea and other skin disorders is only beginning to emerge, and studies have mainly focused on the increase in odds ratio of migraine in patients with rosacea, not the other way around [37, 38]. There are potential pathophysiological overlaps with migraine and neuroendocrineimmune-related skin disorders, in which also calcitonin gene related peptide (CGRP) has been suggested to play a role. Moreover, the role of vascular changes in rosacea and migraine are not fully understood but remain an interesting hypothesis also when reflecting on genetic studies [21, 38,39].

\section{Increased comorbidity based on diagnostic codes and blocks}

The study provided new insight to the migraine related diseasome, and we detected a global holistic increase in frequencies in more abundant diagnostic codes or blocks in migraine patients when compared to controls. The results support previous finding on migraine comorbidity with gastrointestinal, endocrinological, musculosceletal, neurological, and psychiatric disorders as well as with asthma and allergies [9-17, 19, 30]. Moreover, surprisingly many diagnostic codes related to skin, visual and hearing disorders were identified to be more common in migraine. Cardiovascular disorders played a less significant role in the PDNs and only few diagnoses were significantly increased among migraine patients when compared to controls. The reason for the latter may be that the occupational healthcare registry studied here mainly involves outpatient care, and cardiovascular complications are often treated at inpatient care. Increased morbidity of all therapy areas seems to correlate with a recent study by Ziegeler et al. (2019), where they examined all specialists which were consulted due to migraine in an outpatient setting, cardiologists had not been consulted here either, probably due to similar reasons [40].

In addition, several symptoms such as nausea and vomiting, dizziness, malaise and fatigue, and dyspepsia, just to mention a few, were more abundant in migraine when compared to controls. We have previously shown that regardless of increased healthcare resource utilization and prescribed sick-leave days, only less than $10 \%$ were directly linked to migraine diagnoses G43\%. Our findings of increased coexisting morbidities including potential migrainerelated symptoms highlight the difficulty in elucidating the true disease burden directly linked to migraine as it is difficult to determine which diseases and conditions should be included. Regardless, the results of this study provide new insight on a significant and surprisingly holistic multimorbidity related 
to migraine. Whether this is due to a general lower threshold responding to different stimuli that might make migraine patients seek healthcare more often than those without migraine, or due to some other pathophysiological reasons causing increased generalized sensitivity to comorbidities, needs further investigation.

\section{Limitations and strengths}

There are some limitations typically associated with retrospective database analyses, as well as those associated with the cohort selection, many of these have been previously discussed [26]. In addition, real world variation in clinical praxis when examining ICD-10 coded diseasomes cannot be ruled out as subjects may have seen multiple healthcare providers with a variety of specialties. There is thus a risk that some information may not have been consistently recorded for all patients, potentially impacting the population sizes and other outcomes. For example, as we have examined a registry collected as a part of routine clinical practice in an outpatient occupational healthcare setting, some subjects may have additionally visited other healthcare instances e.g. public hospitals for emergency room visits or longerterm specialized healthcare. Another limitation is that as migraine is well known to be an underdiagnosed disease $[26,41,42]$, it is possible that undiagnosed migraine patients in the control group may have confounded the study outcomes.

Notably, the study does not differentiate on disease severity as headache diaries are not yet included in EMRs in the registry. The study is thus based solely on diagnostic codes detected in EMRs, and ICD-10 codes do not separate between episodic and chronic migraine. We have previously reflected on a population receiving and failing prophylaxis for migraine, as having more severe migraine [26]. However, it was not reasonable to include these as a separate group for the diseasome analysis as they only represent $13 \%$ of the examined migraine sample.

Yet, from whatever aspect we chose to examine the morbidity for migraine, the multimorbidity was substantially increased when compared to age- and sex-matched controls without migraine diagnosis. This study, and others, raises questions on comorbidity definition. Is it an increase in frequency of individual diagnoses or should it be based on differences in diagnostic patterns compared to the general population, or as in e.g. genomics, defined by comparing study populations to larger general population samples [43]. Or should more specific methodologies be used to understand disease patterns? In this study we have deliberately taken the decision to discuss about co-existing morbidities and multimorbidity. It is hoped that further methodological and scientific development will take place in evaluating and defining what is really considered as comorbidity.

The strengths of this study partially reflect the limitations, and some of these have been discussed previously [26]. Notably, we have here examined migraine patients truly in a real world setting, and elucidated morbidity of individuals detected as a part of routine clinical praxis reflecting the outcomes resulting from a complex pathophysiological and socio-cultural environment [23]. In addition to previous studies, it is evident that there is substantial multimorbidity in migraine. It may also be important to examine risk patterns of migraine in a timedependent manner. This especially, when morbidities may play a role in migraine chronification [44]. We evaluated the association between comorbidities in PDNs, but not the causality since it would have required directional networks and assessing comorbidities with respect to their timing [25]. Moreover, it is evident that the findings may reflect potentially migraine pathophysiology, drug-related adverse events, and even central sensitisation known to complicate the disease symptomology.

\section{Conclusions}

We have here examined in a holistic and untargeted fashion migraine morbidity. Our data support the previous findings on many of the co-existing morbidities and potential comorbidities for migraine but also brings new insight on the vastness of the morbidity pattern. Regardless of the methodological approach, we detected a holistic increase in multimorbidity among migraine patients when compared to controls across the whole overall ICD-10 coded phenotypic diseasome. Our findings clearly reflect how migraine is observed in a socio-cultural environment and interpreted as a part of routine clinical praxis. As the first untargeted approach to elucidate migraine morbidity, our study may pose a benchmark and a baseline in understanding migraine morbidity detected as ICD-10 codes in the clinical praxis spectrum. More studies are warranted in understanding the pathophysiological causes and consequences for the findings. New migraine treatments may offer a solution in understanding migraine pathophysiology and morbidity patterns through effectiveness analysis focused on the holistic disease burden outcomes in real world settings. 


\section{Appendix 1}

Table 3 Summary of the four reported connectivity measures from the PDNs. Degree, betweenness, closeness, and strength are defined for single diagnosis codes, while modularity is defined for the whole PDN based on a given clustering. For mathematical and formal definitions see [28] and [26]. Note that the $C$ in the value range column stands for the total number of all diagnosis codes in the PDN

\begin{tabular}{|c|c|c|c|}
\hline $\begin{array}{l}\text { Network } \\
\text { measure }\end{array}$ & Calculation & Value range & Interpretation \\
\hline Degree & $\begin{array}{l}\text { Number of phi correlations shared with other diagnosis } \\
\text { codes. }\end{array}$ & $\begin{array}{l}\text { Min: } 2 \text { (or 0) } \\
\text { Max: } C-1\end{array}$ & $\begin{array}{l}\text { Simplest centrality measure that gives a general idea } \\
\text { how involved a diagnosis code is with other diagnosis } \\
\text { codes. Only the directly connected diagnosis codes are } \\
\text { accounted for. *For the analyses in this study, all } \\
\text { diagnosis codes with at most one significant } \\
\text { phi-correlation were excluded, thus the minimum } \\
\text { degree in PDN is } 2 \text {, and not } 0 \text {. }\end{array}$ \\
\hline Strength & $\begin{array}{l}\text { Sum of the phi correlations shared with other diagnosis } \\
\text { codes. }\end{array}$ & $\begin{array}{l}\text { Min: } 0 \\
\text { Max: } C-1\end{array}$ & $\begin{array}{l}\text { Similar to degree but weighted with the phi correlations. } \\
\text { A diagnosis code with a few very strong phi correlations } \\
\text { and a diagnosis code with many weak phi correlations } \\
\text { can have the same strength. } \\
\text { Minimum value = There are no significant phi } \\
\text { correlations. } \\
\text { Maximum value = perfect phi correlation with all other } \\
\text { diagnosis codes. }\end{array}$ \\
\hline Betweenness & $\begin{array}{l}\text { The number of shortest paths between any two diagnosis } \\
\text { codes of which this diagnosis code is a part of divided by } \\
\text { all possible paths. The paths are weighted by the phi } \\
\text { correlations. }\end{array}$ & $\begin{array}{l}\text { Min: } 0 \\
\text { Max: } 1\end{array}$ & $\begin{array}{l}\text { Most diagnosis codes have betweenness } 0 \text {, since most } \\
\text { diagnosis codes are not part of any shortest path [28]. } \\
\text { This measure indicates how central one diagnosis code } \\
\text { is relative to all other diagnosis codes. High } \\
\text { betweenness may indicate that the diagnosis code } \\
\text { mediates the correlation between other diagnosis } \\
\text { codes, leads to many other diagnosis codes, or is the } \\
\text { end point of many comorbidities. }\end{array}$ \\
\hline Closeness & $\begin{array}{l}\text { The inverse of the total sum of the lengths of the shortest } \\
\text { paths to all other diagnosis codes. The path lengths are } \\
\text { weighted by the phi correlations. }\end{array}$ & $\begin{array}{l}\text { Min: } 0 \\
\text { Max: } 1\end{array}$ & $\begin{array}{l}\text { The less intermediary diagnosis codes there are } \\
\text { between two diagnosis codes and the higher the phi } \\
\text { correlations, the 'closer' the two diagnosis codes are. } \\
\text { Closeness gives a measure of this for one diagnosis } \\
\text { code averaged over all other diagnosis codes. High } \\
\text { closeness of a given diagnosis code indicates that it } \\
\text { is more central relative to the other diagnosis codes } \\
\text { and may represent a clinically important aspect of } \\
\text { the diseasome. } \\
\text { Minimum value = no shortest paths lead through the } \\
\text { diagnosis code. } \\
\text { Maximum value = all shortest paths go through the } \\
\text { diagnosis code and all shortest paths have length } 1 .\end{array}$ \\
\hline Modularity & $\begin{array}{l}\text { Number of phi correlations within a cluster divided by the } \\
\text { number of all phi correlations minus the expected proportion } \\
\text { of phi correlations within a random cluster. }\end{array}$ & $\begin{array}{l}\text { Min: }-1 \\
\text { Max: } 1\end{array}$ & $\begin{array}{l}\text { Modularity can be used to compare different clustering } \\
\text { methods or clustering of two networks with the same } \\
\text { algorithm. Here used to sanity check the clustering from } \\
\text { the Walktrap-algorithm. } \\
\text { Modularity < 0: the clustering divides the PDN into } \\
\text { subnetworks worse than expected by a random } \\
\text { clustering. } \\
\text { Modularity > 0: the clustering divides the PDN into } \\
\text { subnetworks better than expected by a random } \\
\text { clustering. }\end{array}$ \\
\hline
\end{tabular}




\section{Appendix 2}

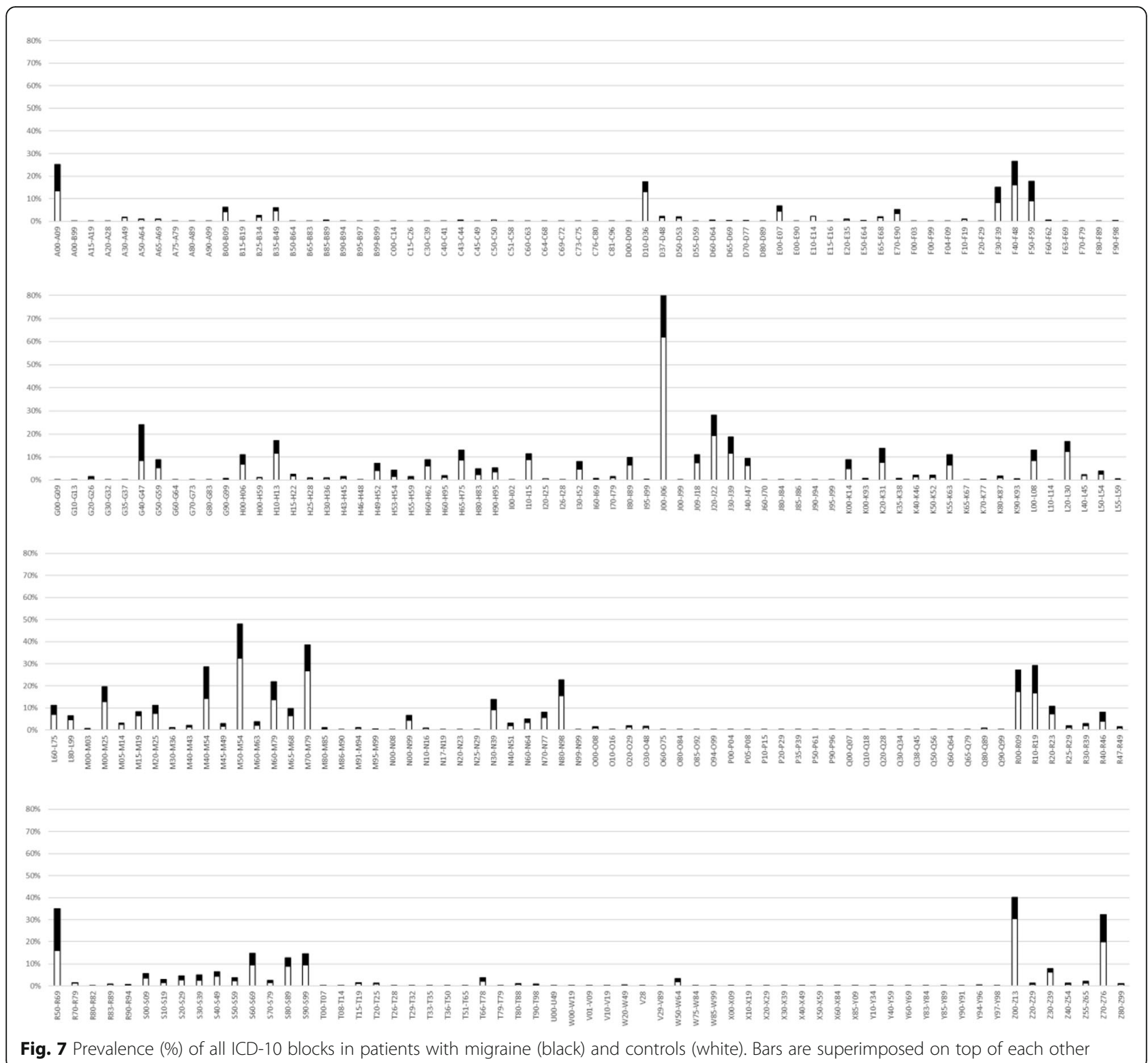

\section{Abbreviations}

EMR: Electronic medical records; PDN: Phenotypic disease networks

\section{Acknowledgements}

The authors wish to thank all co-workers at Novartis, Medaffcon and Terveystalo who have shared their knowledge and support making the study possible.

\section{Authors' contributions}

MAK: contributed to study conception and interpretation of data, was involved in manuscript writing and revision, gave final approval for manuscript for publication, agreed to be accountable for all aspects of the work. TP: contributed to study conception and interpretation of data, was involved in manuscript writing and revision, gave final approval for manuscript for publication, agreed to be accountable for all aspects of the work. JS: contributed to study conception and interpretation of data, was involved in manuscript writing and revision, gave final approval for manuscript for publication, agreed to be accountable for all aspects of the work. SK: contributed to data acquisition and interpretation of data, was involved in manuscript revision, gave final approval for manuscript for publication, agreed to be accountable for all aspects of the work. MN: contributed to data acquisition and interpretation of data, was involved in manuscript revision, gave final approval for manuscript for publication, agreed to be accountable for all aspects of the work. MIL: contributed analysis and interpretation of data, was involved in manuscript writing and revision, gave final approval for manuscript for publication, agreed to be accountable for all aspects of the work. IT: contributed analysis and interpretation of data, was involved in manuscript writing and revision, gave final approval for manuscript for publication, agreed to be accountable for all aspects of the work. ST: contributed analysis and interpretation of data, was involved in manuscript writing and revision, gave final approval for manuscript for publication, agreed to be accountable for all aspects of the work.

\section{Funding}

The study was funded by Novartis Finland Oy. 


\section{Availability of data and materials}

Suomen Terveystalo Plc. authorities are responsible for administrative decisions controlling access to EMR data and ensuring data privacy according the Finnish laws.

\section{Ethics approval and consent to participate}

The use of EMR data of individuals who have given their informed consent at Suomen Terveystalo PIc. has been ethically approved by the National Institute of Health and Welfare in Finland. All patients had given their informed consent.

\section{Consent for publication}

Not applicable.

\section{Competing interests}

MAK, TP and JS are employed by Novartis; SK and MN are employed by Terveystalo; ST, MIL, IT are employed by Medaffcon.

\section{Author details}

${ }^{1}$ Novartis Finland Oy, Espoo, Finland. ${ }^{2}$ Medaffcon Oy, Espoo, Finland.

${ }^{3}$ Terveystalo Biobank Finland, Turku, Finland.

Received: 18 November 2019 Accepted: 23 January 2020

Published online: 31 January 2020

\section{References}

1. Buse DC, Manack AN, Fanning KM, Serrano D, Reed ML, Turkel CC et al (2012 Nov) Chronic migraine prevalence, disability, and Sociodemographic factors: results from the American Migraine Prevalence and Prevention study. Headache J Head Face Pain 52(10):1456-1470

2. D’Amico D, Sansone E, Grazzi L, Giovannetti AM, Leonardi M, Schiavolin S et al (2018 Dec) Multimorbidity in patients with chronic migraine and medication overuse headache. Acta Neurol Scand 138(6):515-522

3. Sinnige J, Korevaar JC, Westert GP, Spreeuwenberg P, Schellevis FG, Braspenning JC (2015 Oct) Multimorbidity patterns in a primary care population aged 55 years and over. Fam Pract 32(5):505-513

4. Vetrano DL, Calderón-Larrañaga A, Marengoni A, Onder G, Bauer JM, Cesar M et al (2018 Sep) An international perspective on chronic multimorbidity: approaching the elephant in the room. J Gerontol A Biol Sci Med Sci 73(10): 1350-1356

5. Lateef T, He J-P, Nelson K, Calkins ME, Gur R, Gur R et al (2019 Feb) Physical-mental comorbidity of pediatric migraine in the Philadelphia neurodevelopmental cohort. J Pediatr 205:210-217

6. Picco L, Achilla E, Abdin E, Chong SA, Vaingankar JA, McCrone P et al (2016) Economic burden of multimorbidity among older adults: impact on healthcare and societal costs. BMC Health Serv Res Available from: https:// www.ncbi.n/m.nih.gov/pmc/articles/PMC4862090/

7. Steiner TJ, Stovner LJ, Vos T, Jensen R, Katsarava Z (2018) Migraine is first cause of disability in under 50s: will health politicians now take notice? J Headache Pain 19(1):17 s10194-018-0846-2

8. Stovner LJ, Nichols E, Steiner TJ, Abd-Allah F, Abdelalim A, Al-Raddadi RM et al (2018) Global, regional, and national burden of migraine and tensiontype headache, 1990-2016: a systematic analysis for the global burden of disease study 2016. Lancet Neurol 17(11):954-976

9. Adelborg K, Szépligeti SK, Holland-Bill L, Ehrenstein V, Horváth-Puhó E, Henderson WW et al (2018) Migraine and risk of cardiovascular diseases: Danish population based matched cohort study. BMJ 31:k96

10. de Tommaso M, Sciruicchio V (2016 Jun 3) Migraine and central sensitization: clinical features, Main comorbidities and therapeutic perspectives. Curr Rheumatol Rev 12(2):113-126

11. Di Stefano M, Pucci E, Miceli E, Pagani E, Brondino N, Nappi G et al (2019 Jun) Prevalence and pathophysiology of post-prandial migraine in patients with functional dyspepsia. Cephalalgia. 15:033310241985759

12. Dresler T, Caratozzolo S, Guldolf K, Huhn J-I, Loiacono C, Niiberg-Pikksööt T et al (2019) Understanding the nature of psychiatric comorbidity in migraine: a systematic review focused on interactions and treatment implications. J Headache Pain 20(1):51

13. Kowalec K, McKay KA, Patten SB, Fisk JD, Evans C, Tremlett H et al (2017 Dec 12) Comorbidity increases the risk of relapse in multiple sclerosis: a prospective study. Neurology. 89(24):2455-2461
14. Luedtke K, Starke W, May A (2018) Musculoskeletal dysfunction in migraine patients. Cephalalgia 38(5):865-875

15. Minen MT (2016) Begasse De Dhaem O, kroon Van Diest a, powers S, Schwedt TJ, Lipton $\mathrm{R}$, et al. migraine and its psychiatric comorbidities. J Neurol Neurosurg Psychiatry 87(7):741-749

16. Moisset X, Bommelaer G, Boube M, Ouchchane L, Goutte M, Dapoigny M et al (2017) Migraine prevalence in inflammatory bowel disease patients: a tertiary-care Centre cross-sectional study. Eur J Pain 21(9):1550-1560

17. Vetvik KG, MacGregor EA (2017 Jan) Sex differences in the epidemiology, clinical features, and pathophysiology of migraine. Lancet Neurol 16(1):76-87

18. Wang S-J (2010) Comorbidities of migraine. Front Neurol 4 Available from: http://journal.frontiersin.org/article/10.3389/fneur.2010.00016/abstract

19. Zarcone D, Corbetta S (2017) Shared mechanisms of epilepsy, migraine and affective disorders. Neurol Sci 38(S1):73-76

20. Harriott AM, Schwedt TJ (2014) Migraine is associated with altered processing of sensory stimuli. Curr Pain Headache Rep 18(11):458

21. van den Maagdenberg AMJM, Nyholt DR, Anttila V (2019) Novel hypotheses emerging from GWAS in migraine? J Headache Pain 20(1):5

22. Xu X, Mishra GD, Jones M (2017) Mapping the global research landscape and knowledge gaps on multimorbidity: a bibliometric study. J Glob Health 7(1):010414

23. Sturmberg JP, Bennett JM, Martin CM, Picard M (2017) 'Multimorbidity' as the manifestation of network disturbances: 'multimorbidity' as network disturbances. J Eval Clin Pract 23(1):199-208

24. Marx P, Antal P, Bolgar B, Bagdy G, Deakin B, Juhasz G (2017) Comorbidities in the diseasome are more apparent than real: What Bayesian filtering reveals about the comorbidities of depression. lakoucheva LM, editor. PLoS Comput Biol 13(6):e1005487

25. Hidalgo CA, Blumm N, Barabási A-L, Christakis NA (2009) A Dynamic Network Approach for the Study of Human Phenotypes. Meyers LA, editor. PLoS Comput Biol 5(4):e1000353

26. Korolainen MA, Kurki S, Lassenius MI, Toppila I, Costa-Scharplatz M, Purmonen T et al (2019) Burden of migraine in Finland: health care resource use, sick-leaves and comorbidities in occupational health care. $J$ Headache Pain 20(1):13

27. Pons P, Latapy M (2005) Computing Communities in Large Networks Using Random Walks. In: Pinar Y, Güngör T, Gürgen F, Özturan C (eds) Computer and Information Sciences - ISCIS 2005. Springer Berlin Heidelberg, Berlin, Heidelberg, pp 284-293 [Cited 2019 Aug 22]. Available from: http://link. springer.com/10.1007/11569596_31

28. Opsahl T, Agneessens F, Skvoretz J (2010) Node centrality in weighted networks: generalizing degree and shortest paths. Soc Networks 32(3): 245-251

29. Charles A (2018) The pathophysiology of migraine: implications for clinical management. Lancet Neurol 17(2):174-182

30. Chen P-K, Wang S-J (2018) Non-headache symptoms in migraine patients. F1000Research 7:188

31. Scher Al, Buse DC, Fanning KM, Kelly AM, Franznick DA, Adams AM et a (2017) Comorbid pain and migraine chronicity: the chronic migraine epidemiology and outcomes study. Neurology 89(5):461-468

32. Akdal G, Dönmez B, Öztürk V, Angın S (2009) Is balance Normal in Migraineurs without history of Vertigo? Headache J Head Face Pain 49(3):419-425

33. Carvalho GF, Almeida CS, Florencio LL, Pinheiro CF, Dach F, Bigal ME et al (2018) Do patients with migraine experience an increased prevalence of falls and fear of falling? A cross-sectional study. Physiotherapy 104(4):424-429

34. Carvalho GF, Bonato P, Florencio LL, Pinheiro CF, Dach F, Bigal ME et al (2017) Balance impairments in different subgroups of patients with migraine. Headache J Head Face Pain 57(3):363-374

35. Rains JC (2018) Sleep and migraine: assessment and treatment of comorbid sleep disorders. Headache J Head Face Pain 58(7):1074-1091

36. Vgontzas A, Pavlović JM (2018) Sleep disorders and migraine: review of literature and potential pathophysiology mechanisms. Headache J Head Face Pain 58(7):1030-1039

37. Christensen CE, Andersen FS, Wienholtz N, Egeberg A, Thyssen JP, Ashina M (2018) The relationship between migraine and rosacea: systematic review and meta-analysis. Cephalalgia 38(7):1387-1398

38. Vera N, Patel NU, Seminario-Vidal L (2018) Rosacea Comorbidities. Dermatol Clin 36(2):115-122 
39. Ashina M, Hansen JM, Do TP, Melo-Carrillo A, Burstein R, Moskowitz MA (2019) Migraine and the trigeminovascular system—40 years and counting. Lancet Neurol 18(8):795-804

40. Ziegeler C, Brauns G, Jürgens TP, May A (2019) Shortcomings and missed potentials in the management of migraine patients experiences from a specialized tertiary care center. J Headache Pain 20(1):86

41. Diamond S, Bigal ME, Silberstein S, Loder E, Reed M, Lipton RB (2006) Patterns of Diagnosis and Acute and Preventive Treatment for Migraine in the United States: Results from the American Migraine Prevalence and Prevention Study. Headache J Head Face Pain 0(0): 061117080657014

42. Tepper SJ, Dahlöf CGH, Dowson A, Newman L, Mansbach H, Jones M et al (2004) Prevalence and diagnosis of migraine in patients consulting their physician with a complaint of headache: data from the landmark study. Headache J Head Face Pain 44(9):856-864

43. International Headache Genetics Consortium, Gormley P, Anttila V, Winsvold BS, Palta P, Esko T et al (2016) Meta-analysis of 375,000 individuals identifies 38 susceptibility loci for migraine. Nat Genet 48(8): 856-866

44. Chalmer MA, Hansen TF, Lebedeva ER, Dodick DW, Lipton RB, Olesen J (2019) Proposed new diagnostic criteria for chronic migraine. Cephalalgia 22:033310241987717

\section{Publisher's Note}

Springer Nature remains neutral with regard to jurisdictional claims in published maps and institutional affiliations.

Ready to submit your research? Choose BMC and benefit from:

- fast, convenient online submission

- thorough peer review by experienced researchers in your field

- rapid publication on acceptance

- support for research data, including large and complex data types

- gold Open Access which fosters wider collaboration and increased citations

- maximum visibility for your research: over $100 \mathrm{M}$ website views per year

At BMC, research is always in progress.

Learn more biomedcentral.com/submissions 